\title{
Mathematical Model in Studying the Stability of Dynamic Systems
}

\author{
Le Hong Lan* \\ Department of Basic Science, Transport and Communications University, \\ No.3, Cau Giay, Hanoi, Vietnam \\ Received 25 April 2017 \\ Revised 30 May 2017; Accepted 05 June 2017
}

\begin{abstract}
In this paper, the author investigated the phenomenon of flutter, which may be the cause of instability of construction structure when it is affected by aerodynamics. By analyzing the effect of aerodynamic on the structure via mathematical analysis, the author has established a mathematical model to study the stability of the structure in the aerodynamic flux that moves supersonically.
\end{abstract}

Keywords: Aerodynamics, flutter, stability.

\section{Introduction}

Aerodynamic load is a significant element when calculating physical structure of large constructions, such as skyscrapers, antenna towers, suspension bridges, etc. This is especially important for flying equipments. Aerodynamic that affects on the structures in both the same direction as well as the perpendicular one of gas flow, depends on magnitudes, characteristics of the gas flow and the movements of the structures. Aerodynamic stability of technical systems is the most important consideration when calculating structures under aerodynamic load. The study of static and dynamic nonlinear patterns of flutter and the aerodynamic stability has a significant impact in practical problems.

Although there are many works dealt with the stability of sheet structures which have composite materials, however, almost the previous publications, the authors have mainly applied finite element methods [1-8]. The main content of this paper is a dynamic analysis of sheet structure that has functionally graded materials under aerodynamic load. One of the unstable situations is flutter, which might cause the instability of structure under aerodynamic load. In the sheet structure the energy from gas flow was generated depending on the movement and speed of such movement. By using nonlinear Piston theory and mathematical analysis, we proposed a qualitative model of nonlinear flutter of structures, and evaluated the stability of the model by numerical analysis.

\footnotetext{
*Corresponding author. Tel.: 84-989060885.

Email: honglanle229@gmail.com

https://doi.org/10.25073/2588-1124/vnumap.4192
} 


\section{Content}

\subsection{The model of nonlinear flutter of sheet structure under aerodynamic load}

Let us investigate a sheet structure from ceramic and metal with the depth $h$, the length $a$ and the width $b$. The outside of the sheet structure is effected by the supersonic gas flow in the parallel direction with the middle surface of the structure. In the coordinate axis $O x y z$, axes $0 x, O y$ and $O z$ describe different directions of the middle surface of the sheet structure.

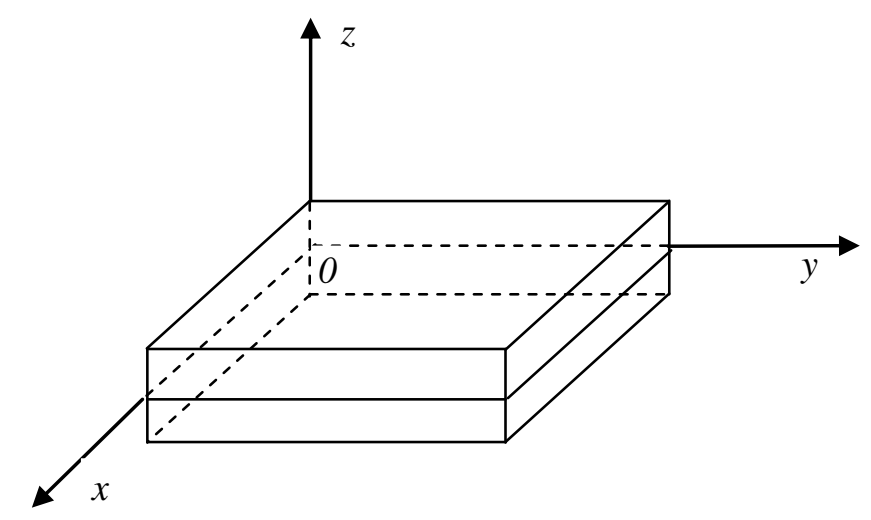

The ratio between the volume of ceramic $V_{c}(z)$ and the one of metal $V_{m}(z)$ is distributed according to the mixed law

$$
V_{c}(z)+V_{m}(z)=1, \quad V_{c}(z)=\left(\frac{2 z+h}{2 h}\right)^{k}
$$

in which, $k \geq 0$ is the volume-fraction index, $z$ is the thickness coordinate of the sheet structure, $z \in\left[-\frac{h}{2}, \frac{h}{2}\right]$. According to the mixed law, module Young $E(z)$ and mass density $\rho(z)$ of the material are expressed under the form

$$
\left\{\begin{array}{l}
E(z)=E_{c} V_{c}+E_{m} V_{m}=E_{m}+\left(E_{c}-E_{m}\right)\left(\frac{2 z+h}{2 h}\right)^{k} \\
\rho(z)=\rho_{c} V_{c}+\rho_{m} V_{m}=\rho_{m}+\left(\rho_{c}-\rho_{m}\right)\left(\frac{2 z+h}{2 h}\right)^{k},
\end{array}\right.
$$

where $E_{c}, E_{m}$ and $\rho_{c}(z), \rho_{m}(z)$ are respectively the module Young and mass densities of the ceramic and of the metal.

The movement of a material point $M(x, y, z)$ in the sheet structure has moving components $u, v$ and $w$ in the directions of $O x, O y$ and $O z$. The transpose in a neighborhood of the point consists of transposed parts that cause the strains and circular motion. The distorted elements are constrained by the conditions that are suitable for the change, in order to ensure the existence of continuous and monotonic solutions.

According to the classical theory of sheet structure with nonlinear geometrical characteristics, socalled Von Korman-Donnell, deformations $\varepsilon(M)=\left(\varepsilon_{x}, \varepsilon_{y}, \gamma_{x y}\right)$ at the point $M(x, y, z)$ having the 
distance $z$ from the middle surface of the sheet structure are presented by transposed elements $u, v, w$ by deformation $\varepsilon^{0}=\left(\varepsilon_{x}^{0}, \varepsilon_{y}^{0}, \gamma_{x y}^{0}\right)$ and by vector curvature of bending sheet $\chi=\left(\chi_{x}, \chi_{y}, \chi_{x y}\right)$ via the relation $\varepsilon(M)=\varepsilon^{0}-z \chi$. Consequently, we have

$$
\varepsilon_{x}=\varepsilon_{x}^{0}-z \chi_{x}, \varepsilon_{y}=\varepsilon_{y}^{0}-z \chi_{y}, \gamma_{x y}=\gamma_{x y}^{0}-2 z \chi_{x y},
$$

where

$$
\begin{cases}\varepsilon_{x}^{0}=\frac{\partial u}{\partial x}+\frac{1}{2}\left(\frac{\partial w}{\partial x}\right)^{2}, & \chi_{x}=\frac{\partial^{2} w}{\partial x^{2}} \\ \varepsilon_{y}^{0}=\frac{\partial v}{\partial y}+\frac{1}{2}\left(\frac{\partial w}{\partial y}\right)^{2}, & \chi_{y}=\frac{\partial^{2} w}{\partial y^{2}} \\ \gamma_{x y}^{0}=\frac{\partial u}{\partial y}+\frac{\partial v}{\partial x}+\frac{\partial w}{\partial x} \frac{\partial w}{\partial y}, & \chi_{x y}=\frac{\partial^{2} w}{\partial x \partial y}\end{cases}
$$

Then, the deformation compatibility equation is

$$
\frac{\partial^{2} \varepsilon_{x}^{0}}{\partial y^{2}}+\frac{\partial^{2} \varepsilon_{y}^{0}}{\partial x^{2}}-\frac{\partial^{2} \gamma_{x y}^{0}}{\partial x \partial y}=\left(\frac{\partial^{2} w}{\partial x \partial y}\right)^{2}-\frac{\partial^{2} w}{\partial x^{2}} \frac{\partial^{2} w}{\partial y^{2}} .
$$

Hooke's law describes the relationship between stress and deformation of the structure as follows, with the Poisson's ratio $v$ is assumed to be constant

$$
\sigma_{x}=\frac{E(z)}{1-v^{2}}\left(\varepsilon_{x}+v \varepsilon_{y}\right), \sigma_{y}=\frac{E(z)}{1-v^{2}}\left(\varepsilon_{y}+v \varepsilon_{x}\right), \tau_{x y}=\frac{E(z)}{2(1+v)} \gamma_{x y} .
$$

Components of internal force and moment are calculated through stress components

$$
\begin{aligned}
& \left\{\begin{array}{l}
N_{x}=\int_{-\frac{h}{2}}^{\frac{h}{2}} \sigma_{x} d z=\frac{E_{1}}{1-v^{2}}\left(\varepsilon_{x}^{0}+v \varepsilon_{y}^{0}\right)-\frac{E_{2}}{1-v^{2}}\left(\chi_{x}+v \chi_{y}\right) \\
N_{y}=\int_{-\frac{h}{2}}^{\frac{h}{2}} \sigma_{y} d z=\frac{E_{1}}{1-v^{2}}\left(\varepsilon_{y}^{0}+v \varepsilon_{x}^{0}\right)-\frac{E_{2}}{1-v^{2}}\left(\chi_{y}+v \chi_{x}\right) \\
N_{x y}=\int_{-\frac{h}{2}}^{\frac{h}{2}} \tau_{x y} d z=\frac{E_{1}}{2(1+v)} \gamma_{x y}^{0}-\frac{E_{2}}{1+v} \chi_{x y}, \\
M_{x}=\int_{-\frac{h}{2}}^{\frac{h}{2}} \sigma_{x} z d z=\frac{E_{2}}{1-v^{2}}\left(\varepsilon_{x}^{0}+v \varepsilon_{y}^{0}\right)-\frac{E_{3}}{1-v^{2}}\left(\chi_{x}+v \chi_{y}\right) \\
M_{y}=\int_{-\frac{h}{2}}^{\frac{h}{2}} \sigma_{y} z d z=\frac{E_{2}}{1-v^{2}}\left(\varepsilon_{y}^{0}+v \varepsilon_{x}^{0}\right)-\frac{E_{3}}{1-v^{2}}\left(\chi_{y}+v \chi_{x}\right) \\
M_{x y}=\int_{-\frac{h}{2}}^{\frac{h}{2}} \tau_{x y} z d z=\frac{E_{2}}{2(1+v)} \gamma_{x y}^{0}-\frac{E_{3}}{1+v} \chi_{x y},
\end{array}\right.
\end{aligned}
$$


in which,

$$
\left\{\begin{array}{l}
E_{1}=\int_{-\frac{h}{2}}^{\frac{h}{2}} E(z) d z=\left(E_{m}+\frac{E_{c}-E_{m}}{k+1}\right) h \\
E_{2}=\int_{-\frac{h}{2}}^{\frac{h}{2}} E(z) z d z=\frac{E_{c}-E_{m}}{2(k+1)(k+2)} k h^{2} \\
E_{3}=\int_{-\frac{h}{2}}^{\frac{h}{2}} E(z) z^{2} d z=\left\{\frac{E_{m}}{12}+\left[\frac{1}{k+3}-\frac{1}{k+2}+\frac{1}{4(k+1)}\right]\left(E_{c}-E_{m}\right)\right\} h^{3} .
\end{array}\right.
$$

From (7) it follows that

$$
\left\{\begin{array}{l}
\varepsilon_{x}^{0}=\frac{1}{E_{1}}\left(N_{x}-v N_{y}\right)+\frac{E_{2}}{E_{I}} \chi_{x} \\
\varepsilon_{y}^{0}=\frac{1}{E_{1}}\left(N_{y}-v N_{x}\right)+\frac{E_{2}}{E_{l}} \chi_{x} \\
\gamma_{x y}^{0}=2\left(\frac{1+v}{E_{l}} N_{x y}+\frac{E_{2}}{E_{l}} \chi_{x y}\right)
\end{array}\right.
$$

Combining with (8) we obtain

$$
\left\{\begin{array}{l}
M_{x}=\frac{E_{2}}{E_{l}} N_{x}-\frac{E_{l} E_{3}-E_{2}^{2}}{E_{l}\left(1-v^{2}\right)}\left(\chi_{x}+v \chi_{y}\right) \\
M_{y}=\frac{E_{2}}{E_{l}} N_{y}-\frac{E_{l} E_{3}-E_{2}^{2}}{E_{l}\left(1-v^{2}\right)}\left(\chi_{y}+v \chi_{x}\right) \\
M_{x y}=\frac{E_{2}}{E_{l}} N_{x y}-\frac{E_{l} E_{3}-E_{2}^{2}}{E_{l}\left(1-v^{2}\right)} \chi_{x y} .
\end{array}\right.
$$

To establish mathematical equations of a sheet structure under aerodynamic load, we consider the case that a sheet lies in the same direction with the movement direction of the supersonic gas flow $U$.

The flow affects on the surface of the sheet structure via the pressure $q_{0}$ in the perpendicular direction with the middle layer of the sheet. According to Love's theory, the equations of motion are given by

$$
\begin{gathered}
\left\{\begin{array}{l}
\frac{\partial N_{x}}{\partial x}+\frac{\partial N_{x y}}{\partial y}=\rho_{0} \frac{\partial^{2} u}{\partial t^{2}} \\
\frac{\partial N_{x y}}{\partial x}+\frac{\partial N_{y}}{\partial y}=\rho_{0} \frac{\partial^{2} v}{\partial t^{2}}
\end{array}\right. \\
\frac{\partial^{2} M_{x}}{\partial x^{2}}+2 \frac{\partial^{2} M_{x y}}{\partial x \partial y}+\frac{\partial^{2} M_{y}}{\partial y^{2}}+\frac{\partial}{\partial x}\left(N_{x} \frac{\partial w}{\partial x}+N_{x y} \frac{\partial w}{\partial y}\right)+\frac{\partial}{\partial y}\left(N_{x y} \frac{\partial w}{\partial x}+N_{y} \frac{\partial w}{\partial y}\right)+q_{0}=\rho_{0} \frac{\partial^{2} w}{\partial t^{2}},
\end{gathered}
$$


where $\rho_{0}=\int_{-h / 2}^{h / 2} \rho(z) d z=\left(\rho_{m}+\frac{\rho_{c}-\rho_{m}}{k+1}\right) h$

According to the nonlinear Piston theory, aerodynamic force affecting on the structure is defined by the formula

$$
q_{0}=-\gamma P_{\infty}\left(M \frac{\partial w}{\partial x}+\frac{1}{a_{\infty}} \frac{\partial w}{\partial t}\right)
$$

in which $\gamma$ is the heat capacity of gas, $a_{\infty}$ is the speed of sound, $P_{\infty}$ is the gas pressure without perturbation. With $U$ is the speed of gas flow, $M:=\frac{U}{a_{\infty}}$ is the Mach number, which characterizes the compression strength of the moving gas flow.

Using Volmir's hypothesis, we have $\rho_{0} \frac{\partial^{2} u}{\partial t^{2}} \rightarrow 0, \rho_{0} \frac{\partial^{2} v}{\partial t^{2}} \rightarrow 0$ as $u \ll w, v \ll w$. By choosing a stress function $\varphi$ such that

$$
N_{x}=\frac{\partial^{2} \varphi}{\partial y^{2}}, N_{y}=\frac{\partial^{2} \varphi}{\partial x^{2}}, N_{x y}=-\frac{\partial^{2} \varphi}{\partial x \partial y},
$$

the equations of (12) becomes homogeneous equations.

The equation (13) is transformed into

$$
\frac{\partial^{2} M_{x}}{\partial x^{2}}+2 \frac{\partial^{2} M_{x y}}{\partial x \partial y}+\frac{\partial^{2} M_{y}}{\partial y^{2}}+N_{x} \frac{\partial^{2} w}{\partial x^{2}}+2 N_{x y} \frac{\partial^{2} w}{\partial x \partial y}+N_{y} \frac{\partial^{2} w}{\partial y^{2}}+q_{0}=\rho_{0} \frac{\partial^{2} w}{\partial t^{2}} .
$$

From (5), (10) and (15) we transform the deformation compatibility equation into

$$
\frac{1}{E_{l}} \Delta \Delta \varphi=\left(\frac{\partial^{2} w}{\partial x \partial y}\right)^{2}-\frac{\partial^{2} w}{\partial x^{2}} \frac{\partial^{2} w}{\partial y^{2}}
$$

From (11), (14), (15) and (16) we have the movement equation

$$
\rho_{0} \frac{\partial^{2} w}{\partial t^{2}}+\gamma P_{\infty}\left(M \frac{\partial w}{\partial x}+\frac{1}{a_{\infty}} \frac{\partial w}{\partial t}\right)+\frac{E_{l} E_{3}-E_{2}^{2}}{E_{l}\left(1-v^{2}\right)} \Delta \Delta w+2 \frac{\partial^{2} \varphi}{\partial x \partial y} \frac{\partial^{2} w}{\partial x \partial y}-\frac{\partial^{2} \varphi}{\partial y^{2}} \frac{\partial^{2} w}{\partial x^{2}}-\frac{\partial^{2} \varphi}{\partial x^{2}} \frac{\partial^{2} w}{\partial y^{2}}=0 .
$$

The system of equations (17) and (18) has unknown functions $\varphi$ and $w$ describing the flutter of the structure under aerodynamic force. This system is used to study the nonlinear flutter and dynamic stability of structure.

\subsection{The analysis of nonlinear dynamic models}

Consider the single mixed sheet in each edge, that satisfies the boundary conditions

$$
\left\{\begin{array}{l}
\left.\left(w=M_{x}=N_{y}=N_{x y}\right)\right|_{(x=0, x=a)}=0 \\
\left.\left(w=M_{y}=N_{y}=N_{x y}\right)\right|_{(y=0, y=b)}=0 .
\end{array}\right.
$$

With the amplitudes of fluctuation $f_{l}(t), f_{2}(t)$ of sheet structure, we will find the solutions of the equations (17) and (18) in the form 


$$
w=f_{1}(t) \sin \frac{m \pi x}{a} \sin \frac{n \pi y}{b}+f_{2}(t) \sin \frac{(m+1) \pi x}{a} \sin \frac{n \pi y}{b},
$$

Such that, they satisfy the boundary condition (19) and the initial condition:

$$
f_{1}(t=0)=f_{1}^{(0)}, \dot{f}_{1}(t=0)=f_{1 l}^{(o)}, f_{2}(t=0)=f_{2}^{(0)}, \dot{f}_{2}(t=0)=f_{2 l}^{(0)} .
$$

Solving the equation (17) with the formula of the equation's solution determined by (20), we find a stress function $\varphi$ in the form

$$
\begin{aligned}
\varphi= & \varphi_{1} \cos \frac{2 n \pi y}{b}+\varphi_{2} \cos \frac{2 m \pi x}{a}+\varphi_{3} \cos \frac{2(m+1) \pi x}{a}+\varphi_{4} \cos \frac{(2 m+1) \pi x}{a} \\
& +\varphi_{5} \cos \frac{\pi x}{a}+\varphi_{6} \cos \frac{2 n \pi y}{b} \cos \frac{(2 m+1) \pi x}{a}+\varphi_{7} \cos \frac{2 n \pi y}{b} \cos \frac{\pi x}{a},
\end{aligned}
$$

where

$$
\begin{aligned}
& \varphi_{1}=\frac{E_{1}\left[f_{1}^{2} m^{2}+f_{2}^{2}(m+1)^{2}\right]}{32 n^{2} \lambda^{2}}, \varphi_{2}=\frac{E_{1} f_{1}^{2} n^{2} \lambda^{2}}{32 m^{2}}, \varphi_{3}=\frac{E_{l} f_{2}^{2} n^{2} \lambda^{2}}{32(m+1)^{2}}, \varphi_{4}=\frac{E_{1} f_{1} f_{2} n^{2} \lambda^{2}}{4(2 m+1)^{2}} \\
& \varphi_{5}=\frac{-E_{1} f_{l} f_{2} n^{2} \lambda^{2}}{4}, \quad \varphi_{6}=\frac{-E_{1} f_{1} f_{2} n^{2} \lambda^{2}}{4\left[(2 m+1)^{2}+4 n^{2} \lambda^{2}\right]^{2}}, \quad \varphi_{7}=\frac{E_{1} f_{1} f_{2} n^{2}(2 m+1)^{2} \lambda^{2}}{4\left(1+4 n^{2} \lambda^{2}\right)^{2}} .
\end{aligned}
$$

Using the Galerkin's method for the equation (18), we obtain the system

$$
\left\{\begin{array}{c}
\rho_{0} \frac{a^{4}}{\pi^{4}} \ddot{f}_{1}+\frac{E_{l} E_{3}-E_{2}^{2}}{E_{l}\left(1-v^{2}\right)}\left(m^{2}+n^{2} \lambda^{2}\right)^{2} f_{1}+\frac{\gamma P_{\infty} a^{4}}{\pi^{4}}\left[-f_{2} \frac{4 M m(m+1)}{a(2 m+1)}+\frac{\dot{f}_{1}}{a_{\infty}}\right]+\frac{f_{1} f_{2}^{2} m^{4} \lambda^{4} E_{1}}{16} \\
\times\left[4+\frac{m^{2}(m+1)^{2}}{n^{4} \lambda^{4}}+\frac{(2 m+1)^{4}}{\left(1+4 n^{2} \lambda^{2}\right)^{2}}+\frac{1}{\left[(2 m+1)^{2}+4 n^{2} \lambda^{2}\right]^{2}}\right]+\frac{f_{1}^{3} E_{1}}{16}\left(m^{4}+n^{4} \lambda^{4}\right)=0 \\
\rho_{0} \frac{a^{4}}{\pi^{4}} \ddot{f}_{2}+\frac{E_{l} E_{3}-E_{2}^{2}}{E_{1}\left(1-v^{2}\right)}\left[(m+1)^{2}+n^{2} \lambda^{2}\right]^{2} f_{2}+\frac{\gamma P_{\infty} a^{4}}{\pi^{4}}\left[f_{1} \frac{4 M m(m+1)}{a(2 m+1)}+\frac{\dot{f}_{2}}{a_{\infty}}\right]+\frac{f_{2} f_{1}^{2} n^{4} \lambda^{4} E_{1}}{16} \\
\times\left[4+\frac{m^{2}(m+1)^{2}}{n^{4} \lambda^{4}}+\frac{(2 m+1)^{4}}{\left(1+4 n^{2} \lambda^{2}\right)^{2}}+\frac{1}{\left[(2 m+1)^{2}+4 n^{2} \lambda^{2}\right]^{2}}\right]+\frac{f_{2}^{3} E_{1}}{16}\left[(m+1)^{4}+n^{4} \lambda^{4}\right]=0 .
\end{array}\right.
$$

Then, we have a relation that determines the partial flutter frequency $\omega$ of the sheet structure as below

$$
\left|K-\omega^{2} H\right|=0 \text {, }
$$

with

$$
K=\left[\begin{array}{cc}
\frac{E_{l} E_{3}-E_{2}^{2}}{E_{l}\left(1-v^{2}\right)}\left(m^{2}+n^{2} \lambda^{2}\right)^{2} & \frac{-4 \gamma P_{\infty} a^{3} M m(m+1)}{\pi^{4}(2 m+1)} \\
\frac{4 \gamma P_{\infty} a^{3} M m(m+1)}{\pi^{4}(2 m+l)} & \frac{E_{l} E_{3}-E_{2}^{2}}{E_{l}\left(1-v^{2}\right)}\left[(m+1)^{2}+n^{2} \lambda^{2}\right]^{2}
\end{array}\right] ; H=\left[\begin{array}{cc}
\rho_{0} \frac{a^{4}}{\pi^{4}} & 0 \\
0 & \rho_{0} \frac{a^{4}}{\pi^{4}}
\end{array}\right] .
$$

The system of nonlinear differential equations (24) và (25) describes flutter and the flutter frequency of the structure under aerodynamic force. To evaluate the fitting of the model, we used RK4 method in Matlab to solve and analyze the stability of the system. 


\subsection{Fitting of the model}

To evaluate the reliability of the method, we study the influence of composite elements of a material on the stability of flutter frequency by using numerical analysis.

The system of nonlinear differential equations (24) satisfies the initial condition

$$
f_{1}(0)=10^{-10}, \dot{f}_{1}(0)=0, f_{2}(0)=10^{-10}, \dot{f}_{2}(0)=0 .
$$

Considering the case where geometrical parameters of the structure are assigned values: $E_{c}=$ $380.10^{9} \mathrm{~N} / \mathrm{m}^{2}, \rho_{c}=3800 \mathrm{~kg} / \mathrm{m}^{3}, E_{m}=70.10^{9} \mathrm{~N} / \mathrm{m}^{2}, \rho_{m}=2720 \mathrm{~kg} / \mathrm{m}^{3} ; v=0,3$ and the characterized factors of aerodynamic force: $\gamma=1,4, \quad P_{\infty}=99473,4 \mathrm{~N} / \mathrm{m}^{2}, \quad a_{\infty}=340 \mathrm{~m} / \mathrm{s}$.

The amplitude of oscillation $f_{1}(t), f_{2}(t)$ of sheet structure, that are described as in the Figures 1 , 2, 3, 4 below shows that the mathematical model is suitable to describe the influence of the component elements.

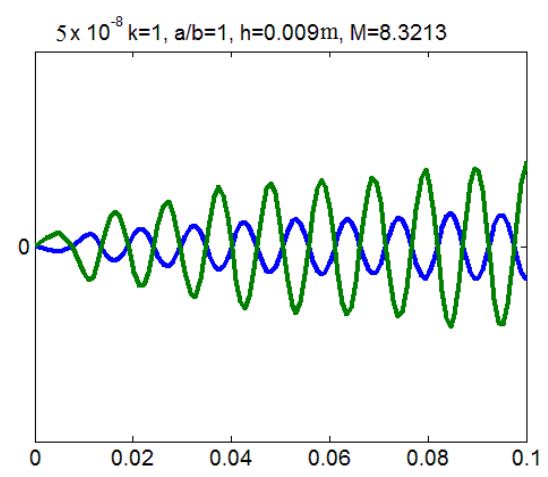

Figure 1. Stable flutter

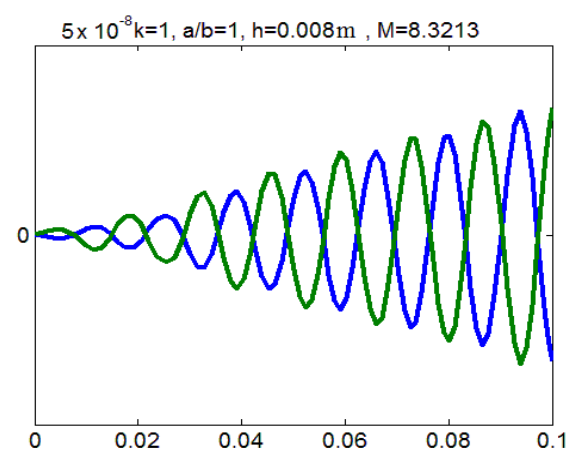

Figure 2. Instable flutter when sheet depth reduces $0,001 \mathrm{~m}$.

With the data described in Figure 1 and Figure 2, we observed that with a small change of the depth of the sheet structure, the flutter will change from a stable state to an unstable one.

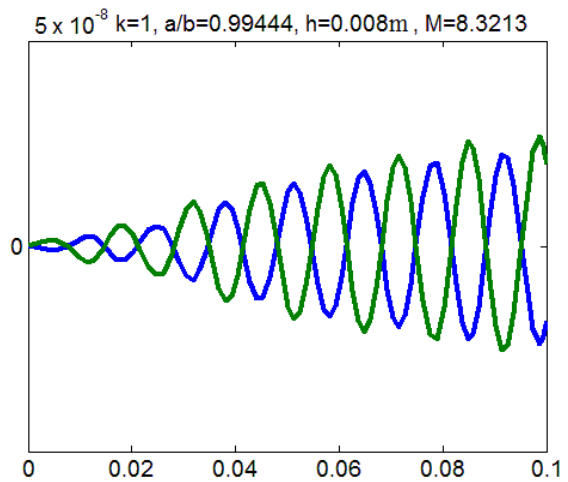

Figure 3. Stable flutter

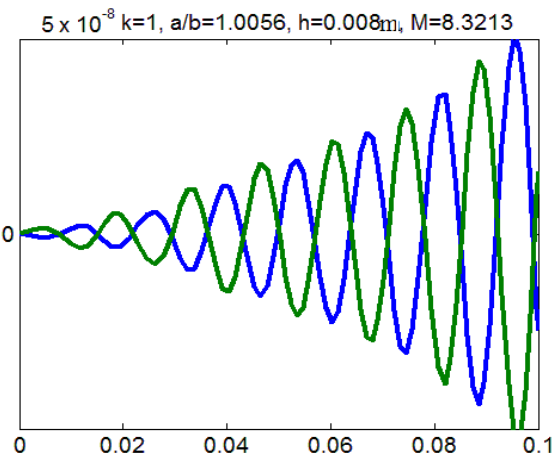

Figure 4. Instable flutter when increasing the ratio $\mathrm{a} / \mathrm{b}$ to 0.01116 .

From the data described in Figure 3 and Figure 4, we see that when there is only a small change of ratio of the sizes of the sheet structure, the flutter will change from a stable state to an unstable state. 


\section{Conclusion}

The mentioned above mathematical model can be used to study the qualitative problems of technical systems of sheet structure of constructions under aerodynamic force. The application of dynamic criteria in investigating the stability of mechanical systems leads to the investigation of the stability of solution of differential equations that describes the motion.

\section{References}

[1] F.Sabri, A.A. Lakis, (2013) Efficient Hybrid Finite Element Method for Flutter Prediction of Functionally Graded Cylindrical Shells, Journal of Vibration and Acoustics 136. First published as doi: 10.1115/1.4025397.

[2] McNamara J.J., Friedmann P.P., Powell K.G., Thuruthimattam B.J. (2005), “ Three- dimensional Aeroelastic and Aerothermoelastic behavior in Hypersonic Flow”, 46 ${ }^{\text {th }}$ AIAA/ASME/ASCE/AHS/ASC Structures, Structural Dynamics \& Material Conference 18-21 April 2005, Texas.

[3] Barbero E. J, Reddy J. N. (1990). "Nonlinear analysic of composite laminated plates using a generalized laminate plate theory”, AIAA Journal, Vol. 28, No.1, pp 1987-1994.

[4] Beldica C. E., Hilton H.H. and Kubair D. (2001). "Viscoelastic panel flutter-stability, probabilities of failure and survival times", Submitted to The $42^{\text {nd }}$ AIAA/ASME/ASCE/AHS/ASC Structures, Structural Dynamics \& Material Conference 16-19 April 2001, Seattle, W.A.

[5] Dey P. and Singha M. K. (2006). "Dynamic stability analysis of composite skew plates subjected to periodic inplane load", Thin-Walled Structures 44, pp. 937 - 942.

[6] Singha M. K and Ganapathi M. (2005). “ A parametric study on supersonic flutter behaviour of laminated composite skew flat panels”, Composite Structures 69, pp. 55-63.

[7] Long N. V., Quoc T. H., Tu T. M. (2016) "Bending and free vibration analysis of functionally graded plates using new eight- unknown shear deformation theory by finite element method", Journal of Science and Technology 54 (3), 402-415.

[8] Bich D. H., Dung D. V., Nam V. H. (2012) "Nonlinear dynamical analysis of eccentrically stiffened functionally graded cylindrical panels", Composite Structures 94(8): 2465-73. 